\title{
ADUBAÇÃO COM NPK EM COQUEIRO ANÃO-VERDE (Cocos nucifera L.) - ATRIBUTOS QUÍMICOS DO SOLO E NUTRIÇÃO DA PLANTA ${ }^{1}$
}

\author{
LUIZ ANTONIO JUNQUEIRA TEIXEIRA², ONDINO CLEANTE BATAGLIA ${ }^{3}$, SALATIÉR BUZETTI ${ }^{4}$, \\ ENES FURLANI JUNIOR ${ }^{4}$
}

\begin{abstract}
RESUMO - Devido à falta de informações sobre adubação de coqueiro para o Estado de São Paulo, realizou-se um experimento de campo em Pereira Barreto $\left(20^{\circ} 47^{\prime} \mathrm{S} ; 51^{\circ} 01^{\prime} \mathrm{W}\right)$, visando a estudar os efeitos da aplicação de NPK em alguns atributos do solo e nutrição da planta, de set/2000 a fev/2004. $\mathrm{O}$ delineamento experimental foi em blocos ao acaso, no esquema fatorial fracionado $1 / 2\left(4^{3}\right)$, com tratamentos constituídos pelas combinações de doses anuais de $\mathrm{N}\left(0 ; 120 ; 240\right.$ e $\left.360 \mathrm{~kg} \mathrm{ha}^{-1} \mathrm{de} \mathrm{N}\right), \mathrm{P}\left(0 ; 100 ; 200\right.$ e $\left.300 \mathrm{~kg} \mathrm{ha}^{-1} \mathrm{de}_{2} \mathrm{O}_{5}\right)$ e K $\left(0 ; 120 ; 240\right.$ e $360 \mathrm{~kg} \mathrm{ha}^{-1}$ de $\left.\mathrm{K}_{2} \mathrm{O}\right)$. As doses de N, Pe K foram aplicadas fracionadamente durante a estação das chuvas, empregando-se como fontes $\mathrm{NH}_{4} \mathrm{NO}_{3}$, superfosfato triplo e $\mathrm{KCl}$, respectivamente. A aplicação de $\mathrm{N}$ em doses superiores a $240 \mathrm{~kg} \mathrm{ha}^{-1}$ ano ${ }^{-1}$ causou excessiva acidificação do solo, sendo que $120 \mathrm{~kg}^{-1}$ ano ${ }^{-1}$ foi suficiente para manter o teor de $\mathrm{N}$ foliar na faixa de suficiência. A adubação com $\mathrm{Pe} \mathrm{K}$ nas doses mais elevadas resultou em perdas por lixiviação. A adubação com $120 \mathrm{~kg}$ ha${ }^{1}$ ano $^{-1}$ de $_{2} \mathrm{O}$ foi suficiente para manter o teor de $\mathrm{K}$ do solo no mesmo nível da amostragem inicial e as plantas com teores de $\mathrm{K}$ foliar acima da faixa de suficiência. O P foliar manteve-se sempre acima da faixa de suficiência, independentemente da adubação fosfatada.
\end{abstract}

Termos para indexação: análise foliar, análise de solo, coco, fósforo, nitrogênio, potássio

\section{NPK FERTILIZATION ON DWARF GREEN COCONUT (Cocos nucifera L.) - SOIL CHEMICAL PROPERTIES AND PLANT NUTRITION}

\begin{abstract}
There are very few information about fertilization of coconut grown in the State of São Paulo, Brazil. So a field experiment with 'Dwarf Green' coconut was carried out to study the effects of NPK rates on some soil chemical properties and plant nutrition, from September, 2000 to February, 2004. The trial was located on an Oxisol in the west part of the State of São Paulo $\left(20^{\circ} 47^{\prime} \mathrm{S} ; 51^{\circ} 01^{\prime} \mathrm{W}\right)$. The experimental design was an incomplete NPK factorial $1 / 2\left(4^{3}\right)$. The treatments were annual rates of $\mathrm{N}\left(0,120,240\right.$, and $360 \mathrm{~kg} \mathrm{ha}^{-1}$ of N), $\mathrm{P}\left(0,100,200\right.$, and $300 \mathrm{~kg} \mathrm{ha}^{-1}$ of $\left.\mathrm{P}_{2} \mathrm{O}_{5}\right)$ and $\mathrm{K}\left(0,120,240\right.$, and $360 \mathrm{~kg} \mathrm{ha}^{-1}$ of $\left.\mathrm{K}_{2} \mathrm{O}\right)$, applied as $\mathrm{NH}_{4} \mathrm{NO}_{3}$, triple superphosphate and $\mathrm{KCl}$, respectively. The rates of $\mathrm{N}$, $\mathrm{Pe} \mathrm{K}$ were split during rainy season. Rates of $\mathrm{N}$ higher than $240 \mathrm{~kg} \mathrm{ha}^{-1} \mathrm{year}^{-1}$ caused excessive soil acidification, whereas $120 \mathrm{~kg} \mathrm{ha}^{-1} \mathrm{year}^{-1}$ was enough to achieve sufficiency range on leaves. Application of higher rates of $\mathrm{P}$ and $\mathrm{K}$ caused losses by leaching. It was necessary $120 \mathrm{~kg} \mathrm{of} \mathrm{K}_{2} \mathrm{O}^{-1} \mathrm{year}^{-1}$ to maintain soil exchangeable $\mathrm{K}$ at the same concentration as found prior to the experiment, the same rate kept leaf $\mathrm{K}$ concentration above sufficiency range. Foliar $\mathrm{P}$ contents were always higher than sufficiency range despite of $\mathrm{P}$ fertilization.
\end{abstract}

Index terms: nitrogen, phosphorus, plant analysis, potassium, soil analysis, coconut.

\section{INTRODUÇÃO}

O cultivo de coco-da-baía no Brasil apresenta duas realidades bastante distintas. De um lado, têm-se os plantios destinados à produção de coco-seco com rentabilidade bastante baixa, o que, nos últimos anos, limitou a expansão dessa atividade e, de outro, as áreas visando à produção de coco-verde, que tiveram grande incentivo devido ao mercado crescente para água de coco. Segundo Saabor et al. (2000), dos 57.000 ha cultivados com coqueiro-anão no Brasil em 2000, cerca de 58\% estariam em fase de formação. Isso implicou oferta crescente de cocoverde nos últimos anos, com significativa diminuição nos preços pagos aos produtores, acentuando a necessidade de se buscarem alternativas para tornar a produção mais eficiente, entre as quais se destaca o manejo adequado da fertilidade do solo.

Entre os nutrientes exportados pelos frutos, destacam-se nitrogênio e potássio. Para uma produção de 119 e 130 cocos por planta, Ouvrier (1984) relatou dados de exportação de 51,72 e 57,05 kg/ha de Ne de 87,46 e 110,39 kg/ha de $\mathrm{K}_{2} \mathrm{O}$. Considerando-se que em áreas irrigadas possam ser colhidos 250 cocos/planta, estima-se que, nessas condições, a remoção de $\mathrm{Ne} \mathrm{K}_{2} \mathrm{O}$ poderia atingir 110 e $190 \mathrm{~kg} / \mathrm{ha}$, respectivamente. Esse aspecto pode comprometer a sustentabilidade dos coqueirais pela remoção de nutrientes e conseqüente esgotamento de suas reservas no solo. Jeganathan (1990), num experimento visando a determinar o efeito da adubação potássica e magnesiana num coqueiral adulto, em três anos consecutivos, atribuiu parte da queda de produção à falta de K. Observou, também, que só havia resposta ao $\mathrm{Mg}$ com suprimento adequado de $\mathrm{K}$.

Santos (1972) reuniu resultados de pesquisa e observações para o Nordeste e elaborou recomendação de adubação, na qual são considerados os teores de $\mathrm{P}$ e $\mathrm{K}$ no solo e a idade das plantas para a determinação das doses de fertilizantes. O acompanhamento do estado nutricional da cultura por meio de análises de tecido foliar foi apontado por Sobral \& Santos (1987) como ferramenta auxiliar para a recomendação de fertilizantes. Entretanto, esses autores não chegaram a detalhar doses em função de teores foliares. Num trabalho mais recente, Sobral (1998) recomenda $3,0 \mathrm{~kg}$ de uréia, $3,2 \mathrm{~kg}$ de superfosfato simples e 2,0 kg de cloreto de potássio, por planta, para coqueiros com 7 anos ou mais, em solos de baixa fertilidade no Nordeste.

Atualmente, a adubação recomendada para a cultura baseia-se na experiência de plantios da região Nordeste, sendo que, no Sudeste, pouco se tem feito para a calibração das doses de N, P e K a serem aplicadas.

O objetivo deste trabalho foi atender à crescente demanda por informações sobre nutrição e adubação de coqueiro e obter dados para subsidiar a elaboração de recomendações de adubação para a cultura nas condições do Estado de São Paulo.

\section{MATERIALEMÉTODOS}

O experimento foi realizado em Pereira Barreto-SP (2047's; $\left.51^{\circ} 01^{\prime} \mathrm{W}\right)$, num Latossolo Vermelho Distrófico numa área de clima tropical úmido com estiagem no inverno (Aw, segundo a classificação de Köppen). O pomar com a variedade anão-verde foi implantado em fevereiro de 1997, sendo que os resultados apresentados neste trabalho foram obtidos entre setembro de 2000 e fevereiro de 2004.

Empregou-se o delineamento experimental de blocos ao acaso, no esquema fatorial fracionado $1 / 2\left(4^{3}\right)$, com tratamentos constituídos pelas combinações de doses anuais de $\mathrm{N}\left(0 ; 120 ; 240\right.$ e $\left.360 \mathrm{~kg} \mathrm{ha}^{-1} \mathrm{de} \mathrm{N}\right)$,

\footnotetext{
1 (Trabalho 131/2004). Recebido: 08/10/2004. Aceito para publicação: 19/04/2005. Realizado com o apoio da FAPESP (Projeto 00/09417-1).

${ }^{2}$ Pesquisador do Instituto Agronômico - IAC, Cx. Postal 28, 13001-970, Campinas-SP, teixeira@iac.sp.gov.br

${ }^{3}$ Pesquisador do Instituto Agronômico - IAC, bolsista do CNPq, ondino@iac.sp.gov.br

${ }^{4}$ Professores da FEIS/UNESP. Ilha Solteira-SP, sbuzetti@agr.feis.unesp.br; enes@agr.feis.unesp.br
} 
$\mathrm{P}\left(0 ; 100 ; 200\right.$ e $300 \mathrm{~kg} \mathrm{ha}^{-1}$ de $\left.\mathrm{P}_{2} \mathrm{O}_{5}\right)$ e $\mathrm{K}\left(0 ; 120 ; 240\right.$ e $360 \mathrm{~kg} \mathrm{ha}^{-1}$ de $\left.\mathrm{K}_{2} \mathrm{O}\right)$, conforme proposto por Colwell (1978). Cada unidade experimental foi composta por nove plantas, sendo sete consideradas para as avaliações. Entre as parcelas, havia uma linha de plantas como bordadura comum. O espaçamento entre plantas foi de 7,5 x 7,5 x 7,5 m (205 plantas/ha). A irrigação foi por microaspersão, empregando-se dois emissores por planta, com aplicação de aproximadamente $85 \mathrm{~L} /$ planta/dia.

No primeiro ano do experimento (2000-2001), com as plantas tendo três anos de idade, as doses de N, P e K foram fracionadas em duas aplicações anuais, no início (set/out) e meio da estação das chuvas (fev/mar). Nos demais, parcelou-se em três aplicações (out, jan e mar). Distribuíram-se os adubos em torno das plantas numa faixa circular com $1,5 \mathrm{~m}$ de largura a $0,5 \mathrm{~m}$ do caule, sem incorporação. As fontes de $\mathrm{N}, \mathrm{Pe}$ $\mathrm{K}$ foram, respectivamente, $\mathrm{NH}_{4} \mathrm{NO}_{3}(32 \% \mathrm{de} \mathrm{N})$, superfosfato triplo ( $41 \%$ de $\left.\mathrm{P}_{2} \mathrm{O}_{5}\right)$ e $\mathrm{KCl}\left(58 \%\right.$ de $\left.\mathrm{K}_{2} \mathrm{O}\right)$. Em toda a área experimental, aplicaram-se zinco (5 $\mathrm{kg} \mathrm{ha}^{-1}$ de $\left.\mathrm{Zn}\right)$; boro (1 $\left.\mathrm{kg} \mathrm{ha}^{-1} \mathrm{de} \mathrm{B}\right)$ e gesso agrícola $\left(1 \mathrm{Mg} \mathrm{ha}^{-1}\right)$.

Os efeitos dos tratamentos em alguns atributos do solo foram avaliados por meio de três amostragens. Coletaram-se amostras de solo para a caracterização da área experimental (set/00) na área de aplicação dos adubos (projeção da copa) e nas entrelinhas, de duas camadas (0 a $20 \mathrm{~cm}$ e de 20 a $40 \mathrm{~cm}$ ). Em abr/02 e ago/03, foram coletadas amostras compostas ( 18 subamostras) de duas camadas ( 0 a 20 e 20 a $40 \mathrm{~cm}$ ), na área de aplicação dos adubos, em cada parcela. As análises de solo seguiram os métodos descritos por Raij et al. (2001).

O estado nutricional das plantas foi avaliado por meio de amostragens foliares e da água dos frutos (albúmen líquido). Amostras de tecido foliar (folha 14) foram coletadas conforme procedimento descrito por Rognon (1984) em mar/01, fev e abr/02, jan e ago/03 e em fev/04. As folhas foram analisadas quanto aos teores de macro e micronutrientes de interesse agrícola, de acordo com Bataglia et al. (1983). Os frutos para análise de água foram colhidos em ago/02, jun e out/03, com aproximadamente sete meses após a abertura da espata. Na água, analisaram-se os teores de $\mathrm{N}$-amoniacal, $\mathrm{P}$ e K, adaptando-se métodos apresentados por Silva (1999) e por Malavolta et al. (1997).

Os efeitos dos tratamentos foram avaliados empregando-se o teste F. Para as situações nas quais se detectaram efeitos significativos dos tratamentos de adubação $(p<0,10)$, foram ajustadas equações de regressão relacionando doses de $\mathrm{N}, \mathrm{P}$ ou $\mathrm{K}$ com as variáveis-resposta.

\section{RESULTADOSE DISCUSSÃO}

\section{Atributos do solo}

Apesar da pouca idade das plantas (3,5 anos) por ocasião da primeira amostragem de solo (set/00), observou-se, na área com maior influência do sistema radicular, grande poder de extração de nutrientes pelo coqueiro, especialmente $\mathrm{K}$ e Ca (Tabela 1). Verificou-se, também, intensa acidificação na área de projeção da copa onde eram aplicados os fertilizantes. Tais resultados são coerentes com os obtidos por Teixeira \& Silva (2003) para diversos genótipos de coqueiro num Latossolo Vermelho Distrófico, em Bebedouro (SP).

$\mathrm{Na}$ falta de um método adequado para análise de $\mathrm{N}$ no solo, verificou-se o efeito da aplicação de $\mathrm{N}$, principalmente, na diminuição da saturação por bases decorrente da acidificação. A capacidade de acidificar o solo de alguns fertilizantes nitrogenados é conhecida e deriva de reações que produzem $\mathrm{H}^{+}$(nitrificação) e da perda de cátions para camadas mais profundas acompanhando o ânion $\mathrm{NO}_{3}^{-}$(Tisdale et al., 1985). A saturação por bases foi linearmente diminuída pela aplicação de $\mathrm{N}$, detectando-se este efeito até na camada de 20 a $40 \mathrm{~cm}$ (Tabela 2). Em média, cada $100 \mathrm{~kg} \mathrm{ha}^{-1}$ de $\mathrm{N}$ aplicados anualmente causou redução de $6 \%$ no valor de $\mathrm{V}$ na camada de 0 a $20 \mathrm{~cm}$ e de $4 \%$, de 20 a $40 \mathrm{~cm}$. Este efeito acentuou-se ao longo do tempo, sugerindo que áreas com aplicações anuais de $\mathrm{N}$ superiores a $120 \mathrm{~kg} \mathrm{ha}^{-1}$ devam ser monitoradas, especialmente em relação à acidez do solo. Estes resultados são compatíveis com os descritos para outros cultivos no Estado de São Paulo, como bananeira (Saes, 1995; Teixeira et al., 2001), seringueira (Bataglia \& Santos, 1999) e citros (Sanches et al., 1999).

A adubação fosfatada teve efeito significativo nos teores de $P$ disponível no solo (Tabela 2). A menor dose de $\mathrm{P}\left(100 \mathrm{~kg} \mathrm{ha}^{-1} \mathrm{de}_{2} \mathrm{O}_{5}\right)$ foi suficiente para promover seu acúmulo no solo ao longo de três anos (Figura 1). Nos tratamentos sem aplicação de P, não houve redução significativa no teor de fósforo disponível no solo, em relação à situação inicial, nas duas épocas de amostragens. Este fato, associado ao aumento do nutriente no solo em resposta à aplicação da menor dose de $\mathrm{P}$, indica que a exigência de fósforo do coqueiro seja relativamente pequena. Em Bebedouro (SP), Teixeira \& Silva (2003) constataram que, para sete genótipos de coqueiro, $19 \mathrm{mg} \mathrm{dm}^{-3}$ de $\mathrm{P}$ disponível no solo foi suficiente para manter a concentração de $\mathrm{P}$ foliar em níveis adequados. Observou-se

TABELA 1 - Atributos químicos do solo cultivado com coqueiro, amostragem inicial em setembro de 2000 das camadas de solo de 0 a $20 \mathrm{~cm}$ e de 20 a $40 \mathrm{~cm}$.

\begin{tabular}{|c|c|c|c|c|c|c|c|c|}
\hline Amostragem & MO & $\mathrm{pH}\left(\mathrm{CaCl}_{2}\right)$ & $\mathbf{P}_{\text {resina }}$ & $\mathbf{K}^{+}$ & $\mathrm{Ca}^{2+}$ & $\mathrm{Mg}^{2+}$ & $\mathbf{H}+\mathbf{A l}$ & $\bar{V}$ \\
\hline & $\mathrm{g} \mathrm{dm}^{-3}$ & & $\mathrm{mg} \mathrm{dm}^{-3}$ & & $\overline{------1}$ & $\mathrm{~m}^{-3}-\cdots$ & & $\%$ \\
\hline Linha ${ }^{(1)}$ & $12 \mathrm{a}^{(3)}$ & $4,2 \mathrm{a}$ & $5 a$ & $\begin{array}{c}0 \mathrm{a} \\
2,0 \mathrm{a}\end{array}$ & $2 \mathrm{a}$ & $2 \mathrm{a}$ & $28 \mathrm{a}$ & $17 \mathrm{a}$ \\
\hline Entrelinh(ł) & $15 \mathrm{a}$ & $4,9 \mathrm{~b}$ & $4 a$ & $\begin{array}{r}4,2 b \\
20\end{array}$ & $6 b$ & $4 \mathrm{a}$ & $20 \mathrm{~b}$ & $41 b$ \\
\hline Linha & $14 \mathrm{a}$ & $4,2 \mathrm{a}$ & $3 a$ & $1,5 \mathrm{a}$ & $3 a$ & $2 \mathrm{a}$ & $30 a$ & $18 \mathrm{a}$ \\
\hline Entrelinha & $12 \mathrm{a}$ & $4,9 \mathrm{~b}$ & $4 a$ & $2,9 b$ & $6 b$ & $5 a$ & $20 \mathrm{~b}$ & $40 \mathrm{~b}$ \\
\hline
\end{tabular}

(1) Área sob a projeção da copa e com aplicação de adubos; ${ }^{(2)}$ área com pouca influência do sistema radicular e sem aplicação de adubos; ${ }^{(3)}$ médias de uma mesma camada seguidas por letras iguais não diferem entre si, pelo teste $\mathrm{F}(p>0,05)$

TABELA 2 - Efeito da aplicação de N, Pe K (doses em kg ha-1 de N, $\mathrm{P}_{2} \mathrm{O}_{5}$ e $\mathrm{K}_{2} \mathrm{O}$ ) na saturação por bases, $\mathrm{P}$ e $\mathrm{K}$ disponíveis do solo.

\begin{tabular}{|c|c|c|c|c|c|}
\hline \multicolumn{2}{|c|}{ Saturação por bases (V\%) } & \multicolumn{2}{|c|}{$P_{\text {resina }}\left(\mathrm{mg} \mathrm{dm}^{-3}\right)$} & \multicolumn{2}{|c|}{$\mathrm{K}^{+}\left(\mathrm{mmol}_{\mathrm{c}} \mathrm{dm}^{-3}\right)$} \\
\hline Função & Valor $p^{(1)}$ & Função & Valor $p$ & Função & Valor $p$ \\
\hline \multicolumn{6}{|c|}{$\begin{array}{l}-- \text { abril } / 02- \\
0 \text { a } 20 \mathrm{~cm}\end{array}$} \\
\hline$\hat{\mathrm{Y}}=40,71-4,67 \times 10^{-2} \mathrm{~N}$ & $<0,0003$ & $\begin{array}{r}\hat{\mathrm{Y}}=5,97+3,15 \times 10^{-1} \mathrm{P} \\
20 \mathrm{a} 40 \mathrm{~cm}\end{array}$ & $<0,0001$ & $\hat{\mathrm{Y}}=1,11+1,04 \times 10^{-2} \mathrm{~K}$ & $<0,0001$ \\
\hline$(\bar{Y}=23)-n s^{(2)}$ & $>0,1$ & $\hat{\mathrm{Y}}=0,80+6,24 \times 10^{-2} \mathrm{P}$ & $<0,0001$ & $\hat{\mathrm{Y}}=1,31+8,10 \times 10^{-3} \mathrm{~K}$ & $<0,0001$ \\
\hline \multicolumn{6}{|c|}{0 a $20 \mathrm{~cm}$} \\
\hline$\hat{\mathrm{Y}}=48,60-7,31 \times 10^{-2} \mathrm{~N}$ & $<0,0001$ & $\begin{array}{r}\hat{\mathrm{Y}}=5,33+5,15 \times 10^{-1} \mathrm{P} \\
20 \mathrm{a} 40 \mathrm{~cm}\end{array}$ & $<0,0001$ & $\hat{\mathrm{Y}}=1,00+9,83 \times 10^{-3} \mathrm{~K}$ & $<0,0001$ \\
\hline$\hat{\mathrm{Y}}=33,21-3,99 \times 10^{-2} \mathrm{~N}$ & $<0,0001$ & $\hat{\mathrm{Y}}=0,89+1,66 \times 10^{-1} \mathrm{P}$ & $<0,0001$ & $\hat{\mathrm{Y}}=0,81+1,01 \times 10^{-2} \mathrm{~K}$ & $<0,0001$ \\
\hline
\end{tabular}

(1) Probabilidade de erro tipo I associada ao teste $\mathrm{F}$ (significância do modelo); ${ }^{(2)}$ valor entre parêntesis corresponde à média da variável; ajuste não significativo ( $p>0,1$ ). 

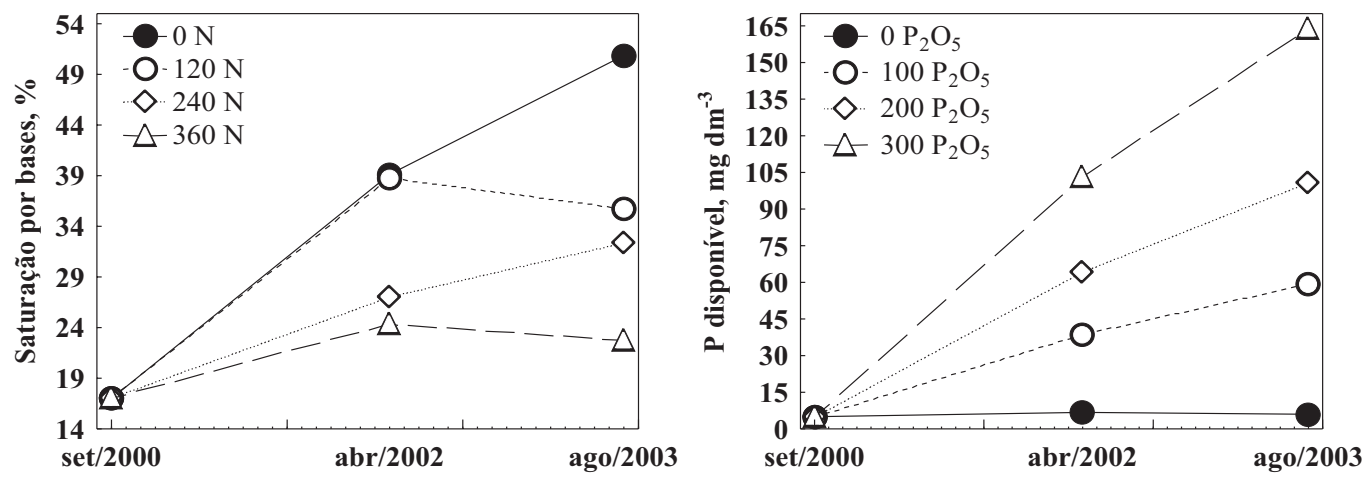

Data da amostragem

Data da amostragem

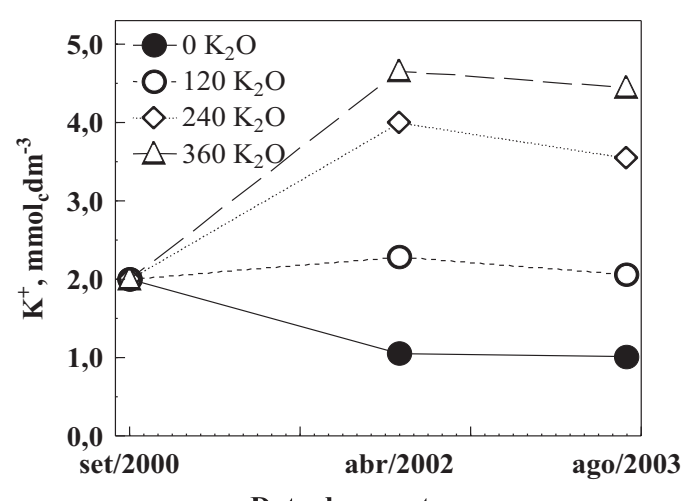

FIGURA 1 - Efeito da aplicação de N, P e K na saturação por bases, P e K ${ }^{+}$disponíveis, respectivamente, na camada de 0 a $20 \mathrm{~cm}$ do solo, em três épocas de amostragem.

que, ao longo de três anos, cada incremento de $100 \mathrm{~kg} \mathrm{ha}^{-1}$ de $\mathrm{P}_{2} \mathrm{O}_{5}$ na aplicação anual implicou, em média, aumento de $41 \mathrm{mg} \mathrm{dm}^{-3}$ no teor de $\mathrm{P}$ disponível na camada de 0 a $20 \mathrm{~cm}$, e de $11 \mathrm{mg} \mathrm{dm}^{-3}$ na de 20 a $40 \mathrm{~cm}$.

A adubação potássica aumentou linearmente os teores de $\mathrm{K}$ do solo (Tabela 2). Cada $100 \mathrm{~kg} \mathrm{ha}^{-1} \mathrm{de}_{2} \mathrm{O}$ aplicados anualmente determinou incremento médio de $1,0 \mathrm{mmol} \mathrm{dm}^{-3}$ na camada de 0 a $20 \mathrm{~cm}$. Ressalte-se o marcante efeito da adubação potássica na lixiviação de $\mathrm{K}$, pois o incremento de $100 \mathrm{~kg} \mathrm{ha}^{-1}$ na dose anual de $\mathrm{K}_{2} \mathrm{O}$ causou aumento médio de $0,9 \mathrm{mmol}$ $\mathrm{dm}^{-3}$ na camada de 20 a $40 \mathrm{~cm}$. Aumento na movimentação de potássio no perfil do solo, proporcional à dose de $\mathrm{K}$ aplicada, também foi descrito por Ritchey (1982) num Latossolo Vermelho-Escuro. Considerando o teor de $\mathrm{K}$ disponível na camada de 0 a $20 \mathrm{~cm}$ obtido na primeira amostragem, estima-se que foi necessário aplicar anualmente cerca de $120 \mathrm{~kg} \mathrm{ha}^{-1} \mathrm{de}$ $\mathrm{K}_{2} \mathrm{O}$ para manter o nível inicial ao longo de três anos (Figura 1).

Em geral, os efeitos da aplicação de N, P e K observados neste experimento coincidem com os descritos por Joseph \& Wahd (1997), que estudaram as alterações em vários atributos de um solo cultivado com coqueiro, durante 22 anos, em resposta à adubação com N, P e K. Destacam- se, entre os aspectos abordados por estes autores, os riscos da acidificação em resposta à aplicação de $\mathrm{N}$; o significativo aumento nos teores de $\mathrm{P}$ e K disponíveis ao longo do tempo, e a recomendação de que aplicações de $\mathrm{Pe}$ $\mathrm{K}$ sejam feitas somente de acordo com as necessidades das plantas detectadas por análise foliar, evitando-se gastos desnecessários.

\section{Estado nutricional}

O estado nutricional das plantas foi monitorado por meio de análises foliares. Observaram-se efeitos relativamente pequenos dos tratamentos nos teores de N, Pe K nas folhas de coqueiro (Tabela 3). Em média, cada aumento de $100 \mathrm{~kg} \mathrm{ha}^{-1} \mathrm{ano}^{-1}$ de $\mathrm{N}$ incrementou o teor de $\mathrm{N}$ foliar em $0,46 \mathrm{~g} \mathrm{~kg}^{-1}$; para $100 \mathrm{~kg} \mathrm{ha}^{-1}$ ano $^{-1} \mathrm{de}_{2} \mathrm{P}_{5}$, a variação foi de $0,05 \mathrm{~g} \mathrm{~kg}^{-1}$ de $\mathrm{Pe}$ a cada $100 \mathrm{~kg} \mathrm{ha}^{-1} \mathrm{ano}^{-1} \mathrm{de} \mathrm{K}_{2} \mathrm{O}$, o aumento (média dos ajustes lineares) foi de $0,92 \mathrm{~g} \mathrm{~kg}^{-1} \mathrm{de} \mathrm{K}$.

Os teores de $\mathrm{N}$ foliar, mesmo sem adubação nitrogenada, mantiveram-se, em parte das amostragens, na faixa de suficiência de 18 a $20 \mathrm{~g} \mathrm{~kg}^{-1}$ de $\mathrm{N}$ para coqueiro-anão apresentada por Magat (2005). Observou-se, também (Figura 2), que cerca de $120 \mathrm{~kg} \mathrm{ha}^{-1}$ de $\mathrm{N}$ foram suficientes para manter o teor foliar na faixa de suficiência.

TABELA 3 - Efeito da aplicação de N, P e K (doses em kg ha-1 de N, $\mathrm{P}_{2} \mathrm{O}_{5}$ e $\mathrm{K}_{2} \mathrm{O}$ ) nos teores de $\mathrm{N}, \mathrm{P}$ e $\mathrm{K}$ em folhas de coqueiro.

\begin{tabular}{|c|c|c|c|c|c|c|}
\hline $\mathrm{N}\left(\mathrm{g} \mathrm{kg}^{-1}\right)$ & \multicolumn{2}{|l|}{$P\left(\mathrm{~g} \mathrm{~kg}^{-1}\right)$} & $K\left(\mathrm{~g} \mathrm{~kg}^{-1}\right)$ & \multicolumn{3}{|c|}{$\mathrm{Cl}\left(\mathrm{g} \mathrm{kg}^{-1}\right)$} \\
\hline Função & Função & $p$ & Função & $p$ & Função & $p$ \\
\hline$=17,88+5,32 \times 10^{-3} \mathrm{~N}<0,0001$ & $(\bar{Y}=1,3)-n s^{(2)}$ & $>0,1$ & $(\bar{Y}=9,9)-n s$ & $>0,1$ & - & - \\
\hline$=17,05+3,01 \times 10^{-3} \mathrm{~N}<0,0029$ & $(\overline{\mathrm{Y}}=1,1)-\mathrm{ns}$ & $>0,1$ & $\hat{\mathrm{Y}}=8,15+7,45 \times 10^{-3} \mathrm{~K}$ & $<0,0032$ & - & - \\
\hline$=19,11+3,39 \times 10^{-3} \mathrm{~N}<0,0013$ & $\hat{\mathrm{Y}}=1,20+4,63 \times 10^{-4} \mathrm{P}$ & $<0,0005$ & $\begin{array}{r}\hat{\mathrm{Y}}=7,80+1,36 \times 10^{-2} \mathrm{~K} \\
--\end{array}$ & $<0,0001$ & $\begin{array}{c}\hat{\mathrm{Y}}=3,75+2,34 \times 10^{-2} \mathrm{~K}- \\
3,62 \times 10^{5} \mathrm{~K}^{2}\end{array}$ & $<0,0001$ \\
\hline$=20,00+2,78 \times 10^{-3} \mathrm{~N}<0,0208$ & $\hat{\mathrm{Y}}=1,16+4,13 \times 10^{-4} \mathrm{P}$ & $<0,0181$ & $\begin{array}{r}\hat{\mathrm{Y}}=11,65+6,68 \times 10^{-3} \mathrm{~K} \\
-\end{array}$ & $<0,0001$ & - & - \\
\hline$=20,54+5,75 \times 10^{-3} \mathrm{~N}<0,0001$ & $\hat{\mathrm{Y}}=1,87+6,25 \times 10^{-4} \mathrm{P}$ & $<0,0001$ & $\begin{array}{l}\hat{\mathrm{Y}}=7,94+1,42 \times 10^{-2} \mathrm{~K}-1,69 \mathrm{x} \\
\text { fevereiro/04 }\end{array}$ & $<0,0001$ & - & - \\
\hline$=17,07+7,67 \times 10^{-3} \mathrm{~N}<0,0001$ & $(\overline{\mathrm{Y}}=1,3)-\mathrm{ns}$ & $>0,1$ & $(\overline{\mathrm{Y}}=10,0)-\mathrm{ns}$ & $>0,1$ & - & - \\
\hline
\end{tabular}

(1) Probabilidade de erro tipo I associada ao teste $\mathrm{F}$ (significância do modelo); ${ }^{(2)}$ valor entre parêntesis corresponde à média da variável; ajuste não significativo $(p>0,1)$.
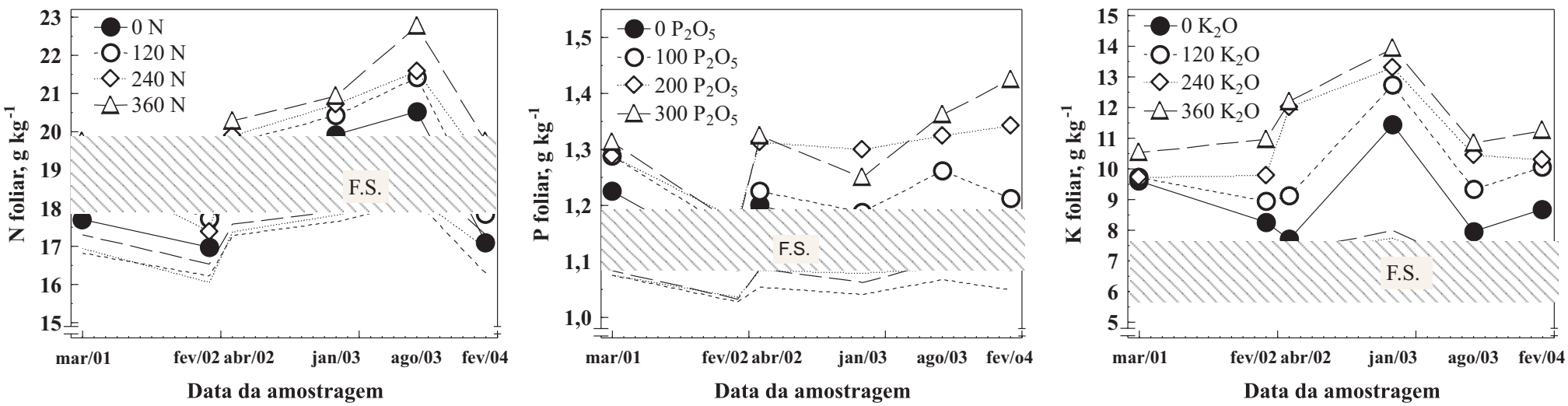

FIGURA 2 - Efeito da aplicação de N, Pe K nos teores de N, Pe K de folhas de coqueiro, respectivamente, em seis épocas de amostragem(F.S. = faixa de suficiência). 
TABELA 4 - Efeito da aplicação de N, Pe K (doses em kg ha ${ }^{-1}$ de N, $\mathrm{P}_{2} \mathrm{O}_{5}$ e $\mathrm{K}_{2} \mathrm{O}$ ) nos teores de N, Pe K na água de coco.

\begin{tabular}{|c|c|c|c|c|c|}
\hline \multicolumn{2}{|c|}{$\mathrm{N}-\mathrm{NH}_{4}\left(\mathrm{mg} \mathrm{L}^{-1}\right)$} & \multicolumn{2}{|l|}{$\mathbf{P}\left(\mathrm{mg} \mathrm{L}^{-1}\right)$} & \multicolumn{2}{|l|}{$K\left(\mathrm{mg} \mathrm{L}^{-1}\right)$} \\
\hline Função & Valor $p^{(1)}$ & Função & Valor $p$ & Função & Valor $p$ \\
\hline$(\bar{Y}=19,8)-n s^{(2)}$ & $>0,1$ & $\hat{\mathrm{Y}}=73,38+4,01 \times 10^{-2} \mathrm{P}$ & $<0,0419$ & $\hat{\mathrm{Y}}=195,62+5,06 \times 10^{-2} \mathrm{~K}$ & $<0,0255$ \\
\hline$(\bar{Y}=36,1)-\mathrm{ns}$ & $>0,1$ & $\hat{\mathrm{Y}}=65,63+3,89 \times 10^{-2} \mathrm{P}$ & $<0,0003$ & $\hat{\mathrm{Y}}=208,72+5,53 \times 10^{-2} \mathrm{~K}$ & $<0,0018$ \\
\hline$(\bar{Y}=20,4)-n s$ & $>0,1$ & $\hat{\mathrm{Y}}=64,71+1,94 \times 10^{-1} \mathrm{P}-4,68 \times 10^{-4} \mathrm{P}^{2}$ & $<0,0059$ & $\hat{\mathrm{Y}}=180,66+1,03 \times 10^{-1} \mathrm{~K}$ & $<0,0340$ \\
\hline
\end{tabular}

(1) Probabilidade de erro tipo I associada ao teste $\mathrm{F}$ (significância do modelo); ${ }^{(2)}$ valor entre parêntesis corresponde à média da variável; ajuste não significativo $(p>0,1)$.

A resposta à adubação com $P$ foi pequena $\mathrm{e}$, independentemente da dose, o teor de $\mathrm{P}$ foliar sempre esteve na faixa de suficiência (Figura 2). Mesmo sem aplicação de $P$, estimou-se que o teor foliar deste nutriente foi superior a $1,1 \mathrm{~g} \mathrm{~kg}^{-1}$ (Tabela 3). A resposta à aplicação de $\mathrm{P}$ e o efeito desta adubação no teor de P disponível do solo ratificam as observações de Khan et al. (1985), que classificaram a cultura como pouco exigente em fósforo.

Observou-se resposta à adubação potássica na maioria das amostragens de tecido foliar (Tabela 3). Entretanto, a concentração de K nas folhas, mesmo nos tratamentos sem adubação potássica, foi quase sempre superior à faixa de suficiência para coqueiro-anão (Figura 2). Plantas bem nutridas em $\mathrm{K}$, mesmo com teor de $\mathrm{K}$ disponível relativamente baixo (em torno de $1,0 \mathrm{mmol}_{\mathrm{c}} \mathrm{dm}^{-3}$ ), indicam que o coqueiro-anão é bastante eficiente na acumulação deste nutriente.

O adequado suprimento de cloro para o coqueiro é importante diante da exigência da cultura, podendo ocorrer deficiência especialmente em cultivos distantes da orla marítima (Uexküll, 1985). Como a necessidade de potássio também é elevada, a aplicação de $\mathrm{KCl}$ serve para atender a estas duas demandas. Observou-se resposta positiva dos teores de potássio e de cloro (Tabela 3) à aplicação de KCl. Entretanto, mesmo sem aplicar cloreto, estimou-se que as plantas estavam suficientemente nutridas com $\mathrm{Cl}$, pois seu teor foliar foi superior ao nível crítico $(2,5 \mathrm{~g} \mathrm{~kg}$ 1', segundo Uexküll, 1985).

Em agosto de 2002, junho e outubro de 2003, analisaram-se os teores de $\mathrm{N}$ amoniacal $\left(\mathrm{N}-\mathrm{NH}_{4}\right)$, P e K na água dos cocos, detectando-se respostas significativas aos tratamentos. Nas três amostragens, os efeitos da aplicação de $\mathrm{P}$ e K na concentração destes nutrientes na água foram positivos (Tabela 4). Em média, cada aumento de $100 \mathrm{~kg} \mathrm{ha}^{-1}$ na dose de $\mathrm{P}_{2} \mathrm{O}_{5}$ implicou incremento de aproximadamente $4,0 \mathrm{mg} \mathrm{L}^{-1}$ no teor de $\mathrm{P}$ da água (média dos ajustes lineares). Para potássio, a cada $100 \mathrm{~kg} \mathrm{ha}^{-1} \mathrm{de}$ $\mathrm{K}_{2} \mathrm{O}$, a variação no teor de $\mathrm{K}$ na água foi de $7,0 \mathrm{mg} \mathrm{L}^{-1}$. Como os efeitos da adubação nos teores de $\mathrm{P}$ e $\mathrm{K}$ foram significativos e consistentes em todas as amostragens, a análise química da água talvez possa tornar-se ferramenta auxiliar no diagnóstico nutricional da cultura, visto que o processamento de amostras de água é mais fácil do que o de amostras de tecido foliar. Jeganathan (1990) também constatou a possibilidade de empregar a análise da água no diagnóstico nutricional da cultura, bem como informou que, em Sri Lanka e em outros locais, este tipo de análise é utilizado com relativo sucesso.

\section{CONCLUSÕES}

1. Apesar da baixa sensibilidade, a análise foliar possibilitou avaliar os efeitos principais e secundários das adubações na nutrição dos coqueiros.

2. A dose de $120 \mathrm{~kg} \mathrm{ha}^{-1} \mathrm{ano}^{-1}$ de $\mathrm{N}$ foi suficiente para manter o teor de $\mathrm{N}$ foliar na faixa de suficiência, sendo que doses superiores a 240 $\mathrm{kg} \mathrm{ha}^{-1} \mathrm{ano}^{-1}$ de N implicaram excessiva acidificação do solo.

3. A adubação com $120 \mathrm{~kg} \mathrm{ha}^{-1}$ ano $^{-1}$ de $\mathrm{K}_{2} \mathrm{O}$ foi suficiente para manter o teor de $\mathrm{K}$ do solo no mesmo nível da amostragem inicial e as plantas com teores de $\mathrm{K}$ foliar acima da faixa de suficiência.

4. As respostas à adubação fosfatada foram pequenas. Independentemente da aplicação de fósforo, o teor de $\mathrm{P}$ foliar sempre esteve acima da faixa de suficiência.
5. A aplicação de doses de P e K mais elevadas causou movimentação destes nutrientes no perfil do solo com alteração na composição da camada subsuperficial, indicando possíveis perdas por lixiviação.

\section{AGRADECIMENTOS}

Os autores agradecem ao Sr. Fancisco Xavier D'Elia, proprietário da área onde foi realizado o experimento, e à FAPESP (Projeto $\mathrm{n}^{\circ}$ 00/ 09417-1), pelo apoio concedido; aos Eng. Agrônomos Carlos A. Pereira e Carlos A. de Morais D'Elia, pelo incentivo à realização do trabalho e apoio nas atividades de campo.

\section{REFERÊNCIAS}

BATAGLIA, O.C.; FURLANI, A.M.C.; TEIXEIRA, J.P.F.; FURLANI, P.R.; GALLO, J.R. Métodos de análise química de plantas. Campinas: IAC, 1983.48p. (Boletim Técnico, 78).

BATAGLIA, O.C.; SANTOS, W.R. Efeitos da adubação NPK na fertilidade do solo, nutrição e crescimento da seringueira. Revista Brasileira de Ciência do Solo, Viçosa, v.23, p.881-890, 1999.

COLWELL, J.D. Computations for studies of soil fertility and fertilizer requirements. Slough: Commonwealth Agricultural Bureaux, 1978. 297p.

JEGANATHAN, M. Studies on potassium-magnesium interaction in coconut (Cocos nucifera).In: Van Beusichem, M.L. (Ed.). Plant nutrition: physiology and applications. Wageningen: Kluwer Academic Publisher, 1990. p.611-77.

JOSEPH, J.; WAHD, P.A. Dynamics of soil nutrient reserves in coconut rhizosphere as influenced by long term inorganic fertilization. Journal of Plantation Crops, Kasarogod, v.25, n.1, p.44-51, 1997.

KHAN, H.H.; BIDDAPPA, C.C.; JOSHI, O.P. A review of Indian work on phosphorus nutrition of coconut. Journal of Plantation Crops, Kasarogod, v.13, n.1, p.11-21, 1985.

MAGAT, S.S. Coconut. In: World fertilizer use manual. International Fertilizer Association (IFA). 2005. Disponível em: <http:// www.fertilizer.org/ifa/publicat/html/pubman/coconut.htm>. Acesso em: 11 abr. 2005.

MALAVOLTA, E.; VITTI, G.C.; OLIVEIRA, S.A. Avaliação do estado nutricional das plantas: princípios e aplicações. 2. ed. Piracicaba: POTAFOS, 1997.319p.

OUVRIER, M. Exportation par la récolte du cocotier PB-121 em function de la fumure potassique et magnésienne. Oléagineux, Montpellier, v.39, p.263-71, 1984.

RAIJ, B. van; ANDRADE, J.C.; CANTARELLA, H.; QUAGGIO, J.A. (Ed.). Análise química para avaliação da fertilidade de solos tropicais. Campinas: IAC, 2001.285p.

RITCHEY, K.D. O potássio nos oxissolos e ultissolos dos trópicos úmidos. Piracicaba: Instituto da Potassa e Fosfato/Instituto Internacional da Potassa, 1982. 69p. (Boletim Técnico, 7)

ROGNON, F. Cocotier. In: MARTIN-PRÉVEL, P.; GAGNARD, J.; GAUTIER, P. (Ed.) L'analyse végétale dans le contrôle de l'alimentation des plantes tempérées et tropicales. Paris: Tec\&Doc, 1984. p.447-57.

SAABOR, A.; LOPES, L.H.S.; CUNHA, M.M.; FERNANDES, C. Coco- 
verde. Brasília: Ministério da Integração Nacional, 2000. 4p. (FrutiSéries, 3)

SAES, L.A. Resposta da bananeira “nanicão” à calagem na região do Vale do Ribeira, 1995. 82f. Dissertação (Mestrado em Agronomia), Escola Superior de Agricultura Luiz de Queiroz, Universidade de São Paulo, Piracicaba, 1995.

SANCHES, A.C.; SILVA, A.P.; TORMENA, C.A.; RIGOLIN, A.T. Impacto do cultivo de citros em propriedades químicas, densidade do solo e atividade microbiana de um podzólico vermelho-amarelo. Revista Brasileira de Ciência do Solo, Viçosa, v.23, p.91-99, 1999.

SANTOS, Z.G. Adubação da cultura do coco. Aracaju: IPEAL, 1972. 8p. (Série Monografia, 6)

SILVA, F. C. Manual de análises químicas de solos, plantas e fertilizantes. Brasília: EMBRAPA, 1999.370p.

SOBRAL, L.F. Nutrição e adubação do coqueiro. In: FERREIRA, J.M.S.; WARWICK, D.R.N.; SIQUEIRA, L.A. A cultura do coqueiro no Brasil. 2.ed. Aracaju: EMBRAPA-CPATC,1998. p.129-157.
SOBRAL, L.F.; SANTOS, Z.G. Sistema de recomendações de fertilizantes para o coqueiro (Cocos nucifera $\mathrm{L}$.) com base na análise foliar. Brasília: EMBRAPA-DDT, 1987.23p. (Documentos, 7)

TEIXEIRA, L.A.J.; NATALE, W.; RUGGIERO, C. Alterações em alguns atributos químicos do solo decorrentes da irrigação e adubação nitrogenada e potássica em bananeira após dois ciclos de cultivo. Revista Brasileira de Fruticultura, Jaboticabal, v.23, n.3, p.684689, 2001.

TEIXEIRA, L.A.J.; SILVA, J.A.A. Nutrição mineral de populações e híbridos de coqueiro (Cocos nucifera L.) cultivados em Bebedouro (SP). Revista Brasileira de Fruticultura, Jaboticabal, v.25, n.2, p.371374, 2003.

TISDALE, S.L.; NELSON, W.; BEATON, J.D. Soil fertility and fertilizers. $4^{\text {th }}$ ed. New York: Macmillan Publishing Company, 1985. 754p.

UEXKÜLL, H.R. von. Nutrition of plantation crops. In: MUNSON, R.D. (Ed.). Potassium in agriculture. Madison: ASA/CSSA/SSSA, 1985. p.929-59. 